\title{
Towards the 1-loop effective action of type IIB orientifolds
}

\author{
Michael Haack* \\ Arnold-Sommerfeld-Center for Theoretical Physics \\ Ludwig-Maximilians-University Munich \\ Theresienstraße 37, 80333 München, Germany \\ E-mail: Michael.Haack@physik. lmu.de

\section{Jin U Kang} \\ Abdus Salam International Centre for Theoretical Physics \\ Strada Costiera 11, Trieste 34014, Italy \\ \& Department of Physics, Kim Il Sung University \\ RyongNam Dong, TaeSong District, Pyongyang, DPR. Korea \\ E-mail: jkangeictp.it
}

\begin{abstract}
We give a short summary of results that are known about the 1-loop corrected low energy effective action of 4-dimensional toroidal type IIB orientifolds with $\mathscr{N}=1$ supersymmetry. Our focus will be on those results which are needed to determine the Kähler potential for the moduli fields.
\end{abstract}

Corfu Summer Institute 2016 "School and Workshops on Elementary Particle Physics and Gravity" 31 August - 23 September, 2016

Corfu, Greece

${ }^{*}$ Speaker. 


\section{Introduction}

The knowledge of (perturbative) quantum corrections to the low energy effective action can be important in many circumstances in string phenomenology, for instance if there is no effect at all to leading order, i.e. at tree level. A well known example is the no-scale structure of the potential arising for example in type IIB flux compactifications on a warped Calabi-Yau space. In this case, quantum corrections are essential in the attempt to stabilize the Kähler moduli [1, 2, 3, 4]. In general quantum corrections to the low energy effective action increase the possibilities for model building and moduli stabilization, as is well exemplified by the large volume scenario [5]. The corresponding non-supersymmetric vacua only exist if one includes the $\alpha^{\prime}$-correction to the Kähler potential derived in [6]. Obviously, it is then important to include further quantum corrections in order to analyze the actual existence and the "precision phenomenology" of this kind of vacua [7].

We will state our calculational setup in sec. 2 and present our (and earlier) results in sec. 3.

\section{Calculational setup}

In this article we focus on theories with $\mathscr{N}=1$ supersymmetry in four dimensions and our main examples are toroidal type IIB orientifolds with this minimal amount of supersymmetry. Let us start by reminding the reader that the general form of the $\mathscr{N}=1$ supersymmetric low energy Wilsonian effective action in the Einstein-frame is determined by three functions, the superpotential $W$, the gauge kinetic function $f_{a b}$ and the Kähler potential, according to

$$
\begin{aligned}
\frac{\mathscr{L}_{\text {bos }}}{(-g)^{1 / 2}}= & \frac{1}{2 \kappa^{2}} R-K_{, \bar{I}} D_{\mu} \bar{\Phi}^{\bar{I}} D^{\mu} \Phi^{J}-\frac{1}{4} \operatorname{Re}\left(f_{a b}(\Phi)\right) F_{\mu v}^{a} F^{b \mu v} \\
& -\frac{1}{8} \operatorname{Im}\left(f_{a b}(\Phi)\right) \varepsilon^{\mu \nu \rho \sigma} F_{\mu \nu}^{a} F_{\rho \sigma}^{b}-V(\Phi, \bar{\Phi})
\end{aligned}
$$

where

$$
\begin{aligned}
V(\Phi, \bar{\Phi}) & =e^{\kappa^{2} K}\left(G^{\bar{I} J} D_{\bar{I}} \bar{W} D_{J} W-3 \kappa^{2}|W|^{2}\right)+\frac{1}{2} \operatorname{Re}\left(f_{a b}\right) D^{a} D^{b}, \\
D_{J} W & =\partial_{\Phi^{J}} W+\kappa^{2}\left(\partial_{\Phi^{J}} K\right) W .
\end{aligned}
$$

As is well known, the superpotential and the gauge kinetic function are holomorphic functions of the scalar fields and they obey non-renormalization theorems ensuring that they only get nonperturbative corrections $(W)$ or 1-loop and non-perturbative corrections $\left(f_{a b}\right)$. Thus, the most complicated ingredient of the $\mathscr{N}=1$ supersymmetric low energy effective action is typically the (real) Kähler potential. Its perturbative quantum corrections can arise from all loop orders. Its 1-loop correction is the focus of this article and we will exemplify our discussion with results from type IIB orientifolds.

Several different methods have been employed in the past in order to determine quantum corrections to the Kähler potential. One approach makes use of known corrections to the type II theory by truncating terms that are eliminated by the orientifold action, see [6] for an example. Alternatively, one might make use of duality to the heterotic string. An example of this can be found in [8]. In the present article we focus on a third approach, using scattering amplitudes in type I theory. 
We will summarize the results obtained in this way in $[9,10,11]$ and also include some work in progress [12].

It turns out that in order to determine the 1-loop corrected Kähler potential of the (untwisted) moduli fields we need to extract from the string amplitudes the 1-loop correction to

- the kinetic term of the scalars,

- the Einstein-Hilbert term,

- the definition of the field variables in which the $\mathscr{N}=1$ supersymmetry is manifest.

The importance of the first point is intuitively clear. The motivation for points 2 and 3 arises from Weyl-rescaling and from the distinction between the string theory basis for the scalar fields (which is used for the string amplitude calculation) and the field theory basis (in which the $\mathscr{N}=1$ supersymmetry is manifest), cf. [13] for instance. This map between string theory and field theory variables in general can get loop corrections.

Let us be a bit more precise on these points. In order not to overload the notation we focus for the present discussion on a single scalar $\tau=\tau^{(0)}+\delta \tau$ which might for instance be a Kähler modulus. $\tau^{(0)}$ is the field theory variable at lowest order, i.e. at tree level, and $\delta \tau$ denotes its 1-loop correction. From string scattering amplitudes one can extract the terms in the low energy effective action in the form

$$
S=\frac{1}{\kappa^{2}} \int d^{4} x \sqrt{-g}\left[(1+\delta E) \frac{1}{2} R+\left(\tilde{G}^{(0)}+\tilde{G}^{(1)}\right) \partial_{\mu} \tau^{(0)} \partial^{\mu} \tau^{(0)}+\ldots\right],
$$

where $\delta E$ is a 1-loop correction to the Einstein-Hilbert term and $\tilde{G}^{(k)}$ are the tree- and 1-loop contributions to the sigma model metric of $\tau^{(0)}$. In writing down (2.4), we assume that the vertex operator for the metric fluctuations is traceless. Thus at string tree level, where the vertex operators are defined, the resulting action would be in Einstein frame, cf. sec. 16.3 in [14]. However, in type II theory and orientifolds thereof there is a 1-loop correction to this which we denoted by $\delta E$ in (2.4), cf. also [15, 16, 17, 18, 19]. Then one can reach the action in Einstein frame (called the "1-loop corrected Einstein frame" in [16]) by performing a Weyl-rescaling

$$
g_{\mu \nu}^{(E)}=(1+\delta E) g_{\mu v} \equiv \Omega^{-2} g_{\mu \nu},
$$

where $g_{\mu \nu}$ denotes the metric appearing in (2.4). The Weyl-rescaling transforms the Ricci-scalar in $D$-dimensions according to

$$
R=\Omega^{-2}\left(R^{(E)}-2(D-1) g^{(E) \mu v} \nabla_{\mu} \partial_{v} \ln \Omega-(D-2)(D-1) g^{(E) \mu v} \partial_{\mu} \ln \Omega \partial_{v} \ln \Omega\right),
$$

cf. appendix D in [20], for instance. Here $R$ is the Ricci-scalar appearing in (2.4). The second term in (2.6) leads to a total derivative and can be dropped and the third term only contributes a term of higher order in the string coupling than 1-loop. Thus, the only effect of the Weyl-rescaling on the sigma model metric in (2.4) to 1-loop order is that $\tilde{G}^{(0)}$ is multiplied by a factor $\Omega^{2}$.

Moreover, if the map between the string and the field theory basis indeed gets modified at 1-loop to

$$
\tau^{(0)}=\tau-\delta \tau
$$


this would lead to a contribution to the $\tau$-metric at 1-loop order according to

$$
\tilde{G}^{(0)}\left(\tau^{(0)}\right) \partial_{\mu} \tau^{(0)} \partial^{\mu} \tau^{(0)}=\tilde{G}^{(0)}(\tau) \partial_{\mu} \tau \partial^{\mu} \tau-\partial_{\tau} \tilde{G}^{(0)}(\tau) \delta \tau \partial_{\mu} \tau \partial^{\mu} \tau-2 \tilde{G}^{(0)}(\tau)\left(\partial_{\tau} \delta \tau\right) \partial_{\mu} \tau \partial^{\mu} \tau
$$

Now that we know which ingredients we need to determine, let us say a few words about the method that we employed in $[9,10,11]$ in order to determine the 1-loop corrections to the scalar metric and the Einstein-Hilbert term. The aim is to read off these quantities from a 2-point function of the corresponding scalar and of 2 gravitons, respectively. However, it is well known that string scattering amplitudes calculate on-shell S-matrix elements and, thus, 2-point functions for massless fields vanish when imposing momentum conservation. In our calculations we used a trick due to [21, 22] (generalized to the graviton 2-point function in [15]). Concretely, we did not impose momentum conservation in intermediate steps. As argued in [22], the scattered states have to be on-shell for consistency of the calculation. However, momentum conservation is imposed by integrating over the string zero modes and one is allowed to leave this integration until the end of the calculation. Not imposing momentum conservation, one obtains a non-zero Lorentz scalar built from the two momenta, i.e.

$$
p_{1}+p_{2} \neq 0 \Longleftrightarrow \delta \equiv p_{1} \cdot p_{2} \neq 0 .
$$

This allows to read off the sigma model metric of the scalars according to

$$
\left\langle\Phi_{i} \Phi_{j}\right\rangle=G_{i j} \delta+\mathscr{O}\left(\delta^{2}\right)
$$

and the correction to the Einstein-Hilbert term according to

$$
\langle h h\rangle \sim(\delta E) p_{2}^{\mu} \varepsilon_{1 \mu \nu} \eta^{v \lambda} \varepsilon_{2 \lambda \rho} p_{1}^{\rho},
$$

where the $\varepsilon$ s are the polarisation tensors of the gravitons $h$. In [21] this trick was used to calculate the 1-loop contribution to the mass of a scalar charged under an anomalous $U(1)$ (i.e. not for its kinetic term) and the result was checked via a 4-point calculation. Similarly, the result for the scalar kinetic terms of an $\mathscr{N}=2$ theory were checked in [9] via a 4-point function and the 2-point function result for the Einstein-Hilbert term of [15] (again for an $\mathscr{N}=2$ theory) were backed by a 3-point amplitude in [17]. Thus, we are confident enough to apply the trick also for corrections to the scalar sigma model metrics and to the Einstein-Hilbert term in models with $\mathscr{N}=1$ supersymmetry (as in [21]). Admittedly, it would be very worthwhile to check our results with the help of 3- or 4-point amplitudes. We leave this for future work.

\section{Results}

In this section we summarize our results pointing out also prior results in the literature. We always use the $\mathbb{Z}_{6}^{\prime}$-orientifold with twist vector

$$
\vec{v}=\left(\frac{1}{6},-\frac{1}{2}, \frac{1}{3}\right)
$$

as our main example, cf. [23]. The reason is that this model has minimal 4D supersymmetry, i.e. $\mathscr{N}=1$, and it contains both D9- and D5-branes which are wrapped around the third torus of the internal orbifold. 


\subsection{Scalar kinetic term}

Let us begin with our results concerning the scalar kinetic terms. In order to be concrete, we specify to the kinetic term of the Kähler modulus of the third torus, i.e.

$$
\tau=\operatorname{Im}\left(T_{3}\right)
$$

which, at sphere level, is given in terms of the string theory variables according to $[15,13]$

$$
\tau^{(0)} \sim e^{-\Phi \mathscr{V} / 3} .
$$

Here (and in the following), $\Phi$ without any subscript denotes the 10-dimensional dilaton and $\mathscr{V} / 3$ is the volume of the third torus measured with the string frame metric.

At sphere level (including $\alpha^{\prime}$-corrections) the metric appearing in (2.4) is given by

$$
\tilde{G}^{(0)}=-\frac{1}{4\left(\tau^{(0)}\right)^{2}}\left(1+\frac{\zeta(3) \chi}{\mathscr{V}}\right)
$$

where $\mathscr{V}$ is the overall volume of the internal space (again measured with the string frame metric) and $\chi$ is its Euler number ( $\chi=48$ for the $\mathbb{Z}_{6}^{\prime}$-orientifold, cf. [24], for instance). The $\mathscr{N}=2$-sector contributions resulting from the annulus $(\mathscr{A})$, Möbius $(\mathscr{M})$ and Klein-bottle $(\mathscr{K})$ were determined

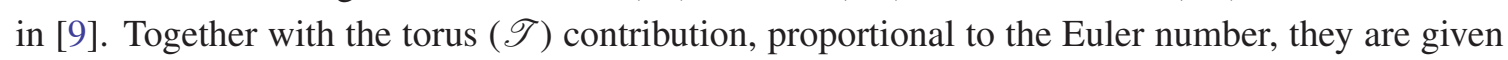
by

$$
\tilde{G}^{(1)} \sim \chi \frac{e^{2 \Phi_{4}}}{\left(\tau^{(0)}\right)^{2}}+a_{1} \frac{1}{\left(\tau^{(0)}\right)^{3} \sigma^{(0)}} E_{2}\left(U_{3}\right)+a_{2} \frac{e^{2 \Phi_{4}} \mathscr{\mathscr { V }} / 2}{\left(\tau^{(0)}\right)^{2}} E_{2}\left(-1 / U_{2}\right) .
$$

Here $\Phi_{4}$ is the 4-dimensional dilaton related to the 10-dimensional one via

$$
e^{-2 \Phi_{4}}=e^{-2 \Phi \mathscr{V}}
$$

$\sigma^{(0)}$ is defined via

$$
\sigma^{(0)}=e^{-\Phi \mathscr{V}},
$$

and $\mathscr{V}_{2}$ is the volume of the second torus and $a_{1}$ and $a_{2}$ are two constants of order 1 that were not determined in [9]. Moreover, $U_{2}$ and $U_{3}$ are the complex structures of the second and third torus, respectively, and the Eisenstein series $E_{2}$ is defined via

$$
E_{2}(U) \equiv \sum_{(m, n) \neq(0,0)} \frac{(\operatorname{Im}(U))^{2}}{|m+n U|^{2}} .
$$

Note that only the complex structure of the second torus (i.e. $U_{2}$ ) is not fixed by the orbifold action.

The new result of [10] was the determination of the $\mathscr{N}=1$ sector contributions of $\mathscr{A}, \mathscr{M}$ and $\mathscr{K}$. Their moduli dependence is simpler than the one of the $\mathscr{N}=2$ sectors. It arises mainly from the normalization of the vertex operators and from the dilaton dependence in Einstein frame. ${ }^{1}$

\footnotetext{
${ }^{1}$ One could expect a more complicated moduli dependence of the $\mathscr{N}=1$ sector contributions in the presence of world volume fluxes (or for branes at angles), interpolating from the corresponding results for gauge thresholds, cf. $[25,26]$. For open string scalars with branes at angles, we found a vanishing result for the $\mathscr{N}=1$ sector contributions of the 1-loop correction to the sigma model metric [27]. It would be interesting to consider the closed string moduli metric for those models as well.
} 
Again for the Kähler modulus of the third torus in the $\mathbb{Z}_{6}^{\prime}$-orientifold, [10] found the result

$$
\tilde{G}_{(\mathscr{N}=1)}^{(1)}=\frac{5}{2^{9} \pi^{3}} \mathrm{Cl}_{2}\left(\frac{\pi}{3}\right) \frac{e^{2 \Phi_{4}}}{\left(\tau^{(0)}\right)^{2}},
$$

where we made use of the second Clausen function

$$
\mathrm{Cl}_{2}(\varphi)=\sum_{k=1}^{\infty} \frac{\sin (k \varphi)}{k^{2}}
$$

and $\mathrm{Cl}_{2}\left(\frac{\pi}{3}\right) \approx 1.015$. Comparing this with (3.5), it is obvious that this represents a correction to the torus contribution (which also arises from the $\mathscr{N}=1$ sector contributions of $\mathscr{T}$ ).

\subsection{Einstein-Hilbert term}

Let us now come to the 1-loop corrections to the Einstein-Hilbert term. In string frame the Einstein-Hilbert term up to 1-loop in Calabi-Yau compactifications of type II theories are known to be of the form [16]

$$
S^{(I I)}=\frac{1}{\kappa^{2}} \int d^{4} x \sqrt{-g}\left[e^{-2 \Phi_{4}}+\frac{\chi}{2 \pi^{3}}\left(\zeta(3) \frac{e^{-2 \Phi_{4}}}{\mathscr{V}} \pm \frac{\pi^{2}}{3}\right)\right] \frac{R}{2}+\ldots,
$$

where the upper (lower) sign corresponds to the result of type IIB (IIA). Obviously, the correction to the Einstein-Hilbert term vanishes on manifolds with $\chi=0$, like $K_{3} \times T^{2}$. Nevertheless, it was shown in [15] that there is a correction to the Einstein-Hilbert term for type I theory even in that case, arising from $\mathscr{A}, \mathscr{M}$ and $\mathscr{K}$. Concretely, they found

$$
S^{(I)}=\frac{1}{\kappa^{2}} \int d^{4} x \sqrt{-g}\left[e^{-2 \Phi_{4}}+a \frac{1}{\mathscr{V}_{T^{2}}} E_{2}(U)\right] \frac{R}{2}+\ldots
$$

for some constant $a$.

In contrast to the situation in type II and type I string theory, there is no 1-loop correction to the Einstein-Hilbert term in 4-dimensional heterotic compactifications [28, 29] and the EinsteinHilbert term only gets $\alpha^{\prime}$-corrections, i.e.

$$
S^{(h e t)}=\frac{1}{\kappa^{2}} \int d^{4} x \sqrt{-g}\left[e^{-2 \Phi_{4}}\left(1+\frac{\chi \zeta(3)}{2 \pi^{3 \mathscr{V}}}\right)\right] \frac{R}{2}+\ldots .
$$

This can be derived in different ways. One possibility is to notice that the integrand of a torus or higher loop graviton 2-point function in heterotic string theory is a total derivative [30]. Another way is to consider the $R^{4}$-terms in 10 dimensions. These come in two different forms, involving different contractions of four Riemann tensors and denoted $t_{8} t_{8} R^{4}$ and $\varepsilon_{10} \varepsilon_{10} R^{4}$ in the literature. We will not need their exact definitions. The only thing we need to know is that the first kind of $R^{4}$ terms leads to a correction of the 4-dimensional kinetic terms of the scalars after compactification on a Calabi-Yau manifold while the second kind leads to a correction of the 4-dimensional EinsteinHilbert term [16]. The $R^{4}$-terms in type II theory were first calculated in $[31,32,33]$ and lead to

$$
\left(\zeta(3) e^{-2 \Phi}+1\right) t_{8} t_{8} R^{4}-\left(\zeta(3) e^{-2 \Phi} \pm 1\right) \varepsilon_{10} \varepsilon_{10} R^{4}
$$

where again the upper (lower) sign refers to type IIB (IIA). Under compactification on a Calabi-Yau manifolds the $\varepsilon_{10} \varepsilon_{10} R^{4}$ term leads to the 4-dimensional Einstein-Hilbert term given in (3.11). 
In contrast the 10 -dimensional $R^{4}$-terms of the heterotic string were determined in $[34,35,36$, $37,38]$ to

$$
\left(\zeta(3) e^{-2 \Phi}+1\right) t_{8} t_{8} R^{4}-\zeta(3) e^{-2 \Phi} \varepsilon_{10} \varepsilon_{10} R^{4} .
$$

Obviously, reducing this to 4 dimensions on a Calabi-Yau manifold does not lead to a 1-loop correction of the Einstein-Hilbert term. An interesting recent discussion about how this is consistent with heterotic / type I duality can be found in [39].

Let us now come to the new result obtained in [11]. Building on earlier work, i.e. [18] and in particular [19] which generalized the method of [15] to the $\mathscr{N}=1$ case, we derived the corrections to the Einstein-Hilbert term for the $\mathbb{Z}_{6}^{\prime}$-orientifold (as well as the $\mathbb{Z}_{3}$-orientifold). We find

$$
\frac{\delta E}{e^{2 \Phi_{4}}}=\frac{\chi}{24 \pi}+\frac{5}{64 \pi^{2}} \mathrm{Cl}_{2}\left(\frac{\pi}{3}\right)-\frac{5}{256 \pi^{2}}\left[\frac{64 \pi^{2} \alpha^{\prime}}{\mathscr{V}_{3}} E_{2}\left(U_{3}\right)-\frac{12 \pi^{2} \alpha^{\prime}}{\mathscr{V} / 2} E_{2}\left(U_{2}\right)-\frac{3 \mathscr{V}_{2}}{4 \pi^{2} \alpha^{\prime}} E_{2}\left(-1 / U_{2}\right)\right]
$$

where the term proportional to the Euler number $\chi$ arise from the torus, while the $\mathrm{Cl}_{2}$-term arises from the $\mathscr{N}=1$ sectors of $\mathscr{A}, \mathscr{M}$ and $\mathscr{K}$ and the $E_{2}$-terms are the contributions of the $\mathscr{N}=2$ sectors of $\mathscr{A}, \mathscr{M}$ and $\mathscr{K}$.

\subsection{Field redefinitions}

Finally let us come to the issue of field redefinitions. This phenomenon is well known in the literature and it is related to the fact that supersymmetry is often not manifest in the string theory field variables for which one can naturally write down the vertex operators (like the volume $\mathscr{V}_{T^{2}}$ of the torus in a $K_{3} \times T^{2}$ compactification, measured in string frame). Rather in order to express the scalar kinetic terms via a Kähler potential or in order to obtain a gauge kinetic function which depends holomorphically on the scalar fields, one usually has to combine the string theory variables to form field theory variables. It is when expressed in terms of these field theory variables that the scalar kinetic terms and the gauge kinetic functions take the form required by supersymmetry. This mapping between string theory and field theory variables often gets modified perturbatively by string loop or $\alpha^{\prime}$ corrections.

For instance, it was observed in [15] that the field theory variable describing the torus volume in a $K_{3} \times T^{2}$ compactification, defined at leading order in (3.3) (with $\mathscr{V}_{3}$ replaced by $\mathscr{V}_{T^{2}}$ ), gets modified to

$$
\tau=\tau^{(0)}+A^{2}+a \frac{E_{2}(U)}{e^{-\Phi \mathscr{V}}}
$$

where $a$ is a constant, $A$ is an open string scalar and $U$ is the complex structure modulus of the torus. In an expansion in the string coupling, the $A^{2}$ - and the $E_{2}$-term appear at a higher order than the leading order term $\tau^{(0)}$. The $A^{2}$-correction was subsequently confirmed to be consistent with the 1-loop calculation of the D-brane gauge couplings in this model [40] and it played a pivotal role in attempts to embed cosmic inflation into string theory [41]. A similar modification of closed string field theory variables due to the presence of an open string sector was discussed in [42, 43], albeit in the presence of non-trivial angles between D-branes or world-volume fluxes, respectively.

Another interesting example can be found in [44], where it was shown that the field theory variables containing the 4-dimensional dilaton and the overall volume of the Calabi-Yau on which 
type II string theory is compactified, get corrections according to

$$
e^{-2 \tilde{\Phi}_{4}}=e^{-2 \Phi_{4}}\left(1+a_{1} \frac{\chi}{\mathscr{V}}+\ldots\right) \quad, \quad \tilde{\mathscr{V}}=\mathscr{V}\left(1+a_{2} \chi e^{2 \Phi_{4}}+\ldots\right)
$$

with constants $a_{1}$ and $a_{2}$.

Finally, a well known example is the perturbative redefinition of the heterotic dilaton field sitting in a chiral $\mathscr{N}=1$ multiplet. This correction can be understood from a perturbative correction to the duality transformation from the dilaton linear multiplet to the chiral multiplet $[45,46,47]$.

For the case at hand, i.e. for the $\mathbb{Z}_{6}^{\prime}$-orientifold, the determination of the field redefinitions and of the final Kähler potential (for the untwisted moduli) is still work in progress [12]. However, we would already like to report on some preliminary results here. Note that one of the scalar fields is naturally described in string theory by a linear multiplet instead of a chiral mulitplet (the analog of the field $S$ in [15], whose axionic scalar is the dual of the RR 2-form $C_{\mu v}$ and whose imaginary part is given by $\operatorname{Im}(S)=e^{-\Phi \mathscr{V}}$ ). Here we do not try to make contact to actual string theory calculations. Rather we assume that we already performed the duality to chiral multiplets only which we denote by $T_{j}=c_{j}+i \tau_{j}\left(j=0, \ldots, 3\right.$ for the untwisted dilaton and Kähler moduli of the $\mathbb{Z}_{6}^{\prime}$-orientifold). All imaginary parts have a leading order part given by $\tau_{j}^{(0)}=e^{-\Phi \mathscr{V}_{j}}$ with $\mathscr{V}_{0} \equiv \mathscr{V}$ and $\mathscr{V}_{i}$ (for $i=1, \ldots, 3)$ being the volume of the $i$ th torus measured with the string frame metric. Moreover, the real parts are given by the dual of $C_{\mu \nu}$ (for $j=0$ ) and by the polarisation of $C_{M N}$ along the $i$ th torus (for $i=1, \ldots, 3$ ).

The $c_{j}$ are axions and enjoy a shift symmetry in string perturbation theory. Of course, this would change under a holomorphic field redefinition of the $T_{j}$, but we work with those field variables for which the shift symmetry is manifest as a symmetry of shifting the real parts of $T_{j}$. This implies that the Kähler potential and, thus, the sigma model metric have to be independent of the $c_{j}$. Consequently, one has

$$
K_{T_{i} \bar{T}_{\bar{j}}} \partial_{\mu} T_{i} \partial^{\mu} \bar{T}_{\bar{j}}=\frac{1}{4} K_{\tau_{i} \tau_{j}}\left(\partial_{\mu} c_{i} \partial^{\mu} c_{j}+\partial_{\mu} \tau_{i} \partial^{\mu} \tau_{j}\right),
$$

i.e.

$$
G_{c_{i} \tau_{j}}=0 \quad, \quad G_{\tau_{i} \tau_{j}}=\frac{1}{4} \frac{\partial^{2} K}{\partial \tau_{i} \partial \tau_{j}}=G_{c_{i} c_{j}} .
$$

Moreover, it turns out that it is sufficient to consider $\tau^{(0)}$-dependent redefinitions of $\tau_{j}^{(0)}$ in order to reach a (shift-symmetric) Kähler metric (if the metric is not already Kähler in terms of the variables $c_{j}$ and $\tau_{j}^{(0)}$ anyway, to begin with). Thus, we only consider

$$
\delta \tau_{i}=\delta \tau_{i}\left(\tau^{(0)}\right) \quad, \quad \delta c_{i}=0
$$

in the following (which is also the only choice that respects the shift symmetry of the real parts of $T_{j}$ ). It turns out that (3.20) and (3.21) together with an assumption about the $\tau$-dependence of the loop corrections to the scalar metric are sufficient to fix the $\tau$-dependence of the field redefinitions and the Kähler potential. More concretely, we assume that the $\tau^{(0)}$-dependence of the 1-loop corrections to the scalar metric takes the form

$$
\mathscr{N}=1: \quad G_{\tau_{i}^{(0)} \tau_{j}^{(0)}}^{(1)}\left(\tau^{(0)}\right)=\alpha_{i j} \frac{e^{2 \Phi_{4}}}{\tau_{i}^{(0)} \tau_{j}^{(0)}}=\frac{\alpha_{i j}}{\tau_{i}^{(0)} \tau_{j}^{(0)} \sqrt{\tau_{0}^{(0)} \tau_{1}^{(0)} \tau_{2}^{(0)} \tau_{3}^{(0)}}}
$$




$$
\mathscr{N}=2: \quad G_{\tau_{i}^{(0)} \tau_{j}^{(0)}}^{(1)}\left(\tau^{(0)}\right)=e^{2 \Phi_{4}} \frac{\sum_{n} \hat{\alpha}_{i j}^{(n)}\left(U_{l}, \mathscr{N}_{l}\right)}{\tau_{i}^{(0)} \tau_{j}^{(0)}}=\frac{\sum_{n} \hat{\alpha}_{i j}^{(n)}\left(U_{l}, \mathscr{V}_{l}\right)}{\tau_{i}^{(0)} \tau_{j}^{(0)} \sqrt{\tau_{0}^{(0)} \tau_{1}^{(0)} \tau_{2}^{(0)} \tau_{3}^{(0)}}}
$$

and similarly for the metric of the $c_{i}$. The $\alpha_{i j}$ in (3.22) are just constants. The sum over $n$ in (3.23) runs over all $\mathscr{N}=2$ sectors. In each of these sectors there is a tower of Kaluza-Klein or winding modes running in the loop which have momentum or winding along the $l$ th torus, respectively. Moreover, the dependence on the volume of the $l$ th torus is $\hat{\alpha}_{i j}^{(n)}\left(U_{l}, \mathscr{V}_{l}\right) \sim \mathscr{V}_{l}^{-1}$ for the contribution of Kaluza-Klein modes and $\hat{\alpha}_{i j}^{(n)}\left(U_{l}, \mathscr{V}_{l}\right) \sim \mathscr{V}_{l}$ for the contribution of winding modes. The dependence on the complex structure $U_{l}$ of the $l$ th torus is left arbitrary. This ansatz is suggested by concrete string theory calculations $[9,7,10]$. Using this ansatz and (3.20) and (3.21) we find for the $\mathbb{Z}_{6}^{\prime}$-orientifold, for the field redefinition of $\tau_{3}$, for instance, ${ }^{2}$

$$
\begin{aligned}
\delta \tau_{3}= & a_{1} \sqrt{\frac{T_{3}^{(0)}-\bar{T}_{3}^{(0)}}{\left(S^{(0)}-\bar{S}^{(0)}\right)\left(T_{1}^{(0)}-\bar{T}_{1}^{(0)}\right)\left(T_{2}^{(0)}-\bar{T}_{2}^{(0)}\right)}} \\
& +a_{2} \frac{\left(T_{3}^{(0)}-\bar{T}_{3}^{(0)}\right) E_{2}\left(U_{2}\right)}{\left(S^{(0)}-\bar{S}^{(0)}\right)\left(T_{2}^{(0)}-\bar{T}_{2}^{(0)}\right)}+a_{3} \frac{E_{2}\left(-1 / U_{2}\right)}{\left(T_{1}^{(0)}-\bar{T}_{1}^{(0)}\right)}+a_{4} \frac{E_{2}\left(U_{3}\right)}{\left(S^{(0)}-\bar{S}^{(0)}\right)}
\end{aligned}
$$

and for the Kähler potential of the dilaton and the untwisted Kähler moduli

$$
\begin{aligned}
K= & -\ln (S-\bar{S})-\ln \left[\left(T_{1}-\bar{T}_{1}\right)\left(T_{2}-\bar{T}_{2}\right)\left(T_{3}-\bar{T}_{3}\right)\right] \\
& -b_{1} \chi \zeta(3) \sqrt{\frac{\left(T_{1}-\bar{T}_{1}\right)\left(T_{2}-\bar{T}_{2}\right)\left(T_{3}-\bar{T}_{3}\right)}{(S-\bar{S})^{3}}} \\
& +b_{2} \frac{E_{2}\left(U_{2}\right)}{\left(T_{2}-\bar{T}_{2}\right)(S-\bar{S})}+b_{3} \frac{E_{2}\left(-1 / U_{2}\right)}{\left(T_{1}-\bar{T}_{1}\right)\left(T_{3}-\bar{T}_{3}\right)}+b_{4} \frac{E_{2}\left(U_{3}\right)}{\left(T_{3}-\bar{T}_{3}\right)(S-\bar{S})} \\
& +b_{5} \frac{1}{\sqrt{\left(T_{1}-\bar{T}_{1}\right)\left(T_{2}-\bar{T}_{2}\right)\left(T_{3}-\bar{T}_{3}\right)(S-\bar{S})}} .
\end{aligned}
$$

All the coefficients $a_{i}$ in (3.24) and $b_{i}$ in (3.25) are constants that have to be determined by comparing to actual string theory calculations.

Here we just want to end with a few comments. The term in the first row of (3.24) arises from $\mathscr{N}=1$-sectors of $\mathscr{A}, \mathscr{M}, \mathscr{K}$ and $\mathscr{T}$, whereas the terms in the second row arise from $\mathscr{N}=2$ sectors of $\mathscr{A}, \mathscr{M}$ and $\mathscr{K}$. The final term (with coefficient $a_{4}$ ) is the analog of the correction of (3.17). It would be interesting to check the 1-loop field redefinition (3.24) also by calculating the D5-brane gauge coupling at 3/2-loop order, i.e. from surfaces with Euler number $\chi=-1$ which are discussed for example in [48]. In the Kähler potential (3.25), the term in the second row represents the $\alpha^{\prime}$-correction from sphere level, the terms in the third row arise from $\mathscr{N}=2$-sectors of $\mathscr{A}, \mathscr{M}$ and $\mathscr{K}$ and the term in the last row has its origin in $\mathscr{N}=1$-sectors of $\mathscr{A}, \mathscr{M}, \mathscr{K}$ and $\mathscr{T}$.

Acknowledgments: M.H. is thankful to Ioannis Bakas and Dieter Lüst for organizing the stimulating workshop at Ringberg Castle. He thanks Dieter Lüst for continuing the organization even after

\footnotetext{
${ }^{2}$ here and in (3.25) we renamed $T_{0} \rightarrow S$ in order to conform with the usual notation in the literature
} 
Ioannis' sudden and sad death. It was a big loss. Ioannis will be remembered for his insightful physics and his kind personality.

J.K. thanks Ignatios Antoniadis, Michele Cicoli, Atish Dabholkar, Edi Gava, Kumar S. Narain, Fernando Quevedo and Giovanni Villadoro for interesting discussions. M.H. thanks Ralph Blumenhagen, Ilka Brunner, Stephan Stieberger and Pierre Vanhove for interesting discussions. Moreover, he is thankful to Fernando Quevedo and the ICTP for their hospitality during part of this work. Many thanks also to Marcus Berg for countless discussions and collaboration on many of the topics presented here. The work of M.H. was supported by the Excellence Cluster "The Origin and the Structure of the Universe" in Munich and by the German Research Foundation (DFG) within the Emmy-Noether-Program (grant number: HA 3448/3-1).

\section{References}

[1] S. Kachru, R. Kallosh, A. D. Linde and S. P. Trivedi, "De Sitter vacua in string theory," Phys. Rev. D 68 (2003) 046005 doi:10.1103/PhysRevD.68.046005 [hep-th/0301240].

[2] G. von Gersdorff and A. Hebecker, "Kähler corrections for the volume modulus of flux compactifications,” Phys. Lett. B 624 (2005) 270 doi:10.1016/j.physletb.2005.08.024 [hep-th/0507131].

[3] M. Berg, M. Haack and B. Körs, “On volume stabilization by quantum corrections,” Phys. Rev. Lett. 96 (2006) 021601 doi:10.1103/PhysRevLett.96.021601 [hep-th/0508171].

[4] M. Cicoli, J. P. Conlon and F. Quevedo, "General Analysis of LARGE Volume Scenarios with String Loop Moduli Stabilisation,” JHEP 0810 (2008) 105 doi:10.1088/1126-6708/2008/10/105 [arXiv:0805.1029 [hep-th]].

[5] V. Balasubramanian, P. Berglund, J. P. Conlon and F. Quevedo, "Systematics of moduli stabilisation in Calabi-Yau flux compactifications,” JHEP 0503 (2005) 007 doi:10.1088/1126-6708/2005/03/007 [hep-th/0502058].

[6] K. Becker, M. Becker, M. Haack and J. Louis, "Supersymmetry breaking and alpha-prime corrections to flux induced potentials,” JHEP 0206 (2002) 060 doi:10.1088/1126-6708/2002/06/060 [hep-th/0204254].

[7] M. Berg, M. Haack and E. Pajer, "Jumping Through Loops: On Soft Terms from Large Volume Compactifications,” JHEP 0709 (2007) 031 doi:10.1088/1126-6708/2007/09/031 [arXiv:0704.0737 [hep-th]].

[8] P. G. Camara and E. Dudas, "Multi-instanton and string loop corrections in toroidal orbifold models," JHEP 0808 (2008) 069 doi:10.1088/1126-6708/2008/08/069 [arXiv:0806.3102 [hep-th]].

[9] M. Berg, M. Haack and B. Körs, "String loop corrections to Kähler potentials in orientifolds," JHEP 0511 (2005) 030 doi:10.1088/1126-6708/2005/11/030 [hep-th/0508043].

[10] M. Berg, M. Haack, J. U. Kang and S. Sjörs, “Towards the one-loop Kähler metric of Calabi-Yau orientifolds," JHEP 1412 (2014) 077 doi:10.1007/JHEP12(2014)077 [arXiv:1407.0027 [hep-th], arXiv:1407.0027].

[11] M. Haack and J. U. Kang, "One-loop Einstein-Hilbert term in minimally supersymmetric type IIB orientifolds,” JHEP 1602 (2016) 160 doi:10.1007/JHEP02(2016)160 [arXiv:1511.03957 [hep-th]].

[12] M. Haack and J. U. Kang, "One-loop field redefinition for Kähler variables in $\mathscr{N}=1$ type IIB orientifolds," work in progress. 
[13] D. Lüst, P. Mayr, R. Richter and S. Stieberger, "Scattering of gauge, matter, and moduli fields from intersecting branes,” Nucl. Phys. B 696 (2004) 205 doi:10.1016/j.nuclphysb.2004.06.052 [hep-th/0404134].

[14] R. Blumenhagen, D. Lüst and S. Theisen, "Basic concepts of string theory," doi:10.1007/978-3-642-29497-6

[15] I. Antoniadis, C. Bachas, C. Fabre, H. Partouche and T. R. Taylor, “Aspects of type I - type II heterotic triality in four-dimensions," Nucl. Phys. B 489 (1997) 160 doi:10.1016/S0550-3213(96)00514-7 [hep-th/9608012].

[16] I. Antoniadis, S. Ferrara, R. Minasian and K. S. Narain, " $R^{4}$ couplings in M and type II theories on Calabi-Yau spaces,” Nucl. Phys. B 507 (1997) 571 doi:10.1016/S0550-3213(97)00572-5 [hep-th/9707013].

[17] I. Antoniadis, R. Minasian and P. Vanhove, "Noncompact Calabi-Yau manifolds and localized gravity,” Nucl. Phys. B 648 (2003) 69 doi:10.1016/S0550-3213(02)00974-4 [hep-th/0209030].

[18] E. Kohlprath, "Induced gravity in Z(N) orientifold models," Nucl. Phys. B 697 (2004) 243 doi:10.1016/j.nuclphysb.2004.06.040 [hep-th/0311251].

[19] F. T. J. Epple, "Induced gravity on intersecting branes," JHEP 0409 (2004) 021 doi:10.1088/1126-6708/2004/09/021 [hep-th/0408105].

[20] R. M. Wald, “General Relativity,” Chicago, Usa: Univ. Pr. ( 1984) 491p doi:10.7208/chicago/9780226870373.001.0001

[21] J. J. Atick, L. J. Dixon and A. Sen, "String Calculation of Fayet-Iliopoulos d Terms in Arbitrary Supersymmetric Compactifications,” Nucl. Phys. B 292 (1987) 109. doi:10.1016/0550-3213(87)90639-0

[22] J. A. Minahan, "One Loop Amplitudes on Orbifolds and the Renormalization of Coupling Constants," Nucl. Phys. B 298, 36 (1988). doi:10.1016/0550-3213(88)90303-3

[23] G. Aldazabal, A. Font, L. E. Ibanez and G. Violero, “D = 4, N=1, type IIB orientifolds,” Nucl. Phys. B 536 (1998) 29 doi:10.1016/S0550-3213(98)00666-X [hep-th/9804026].

[24] R. Blumenhagen, B. Körs, D. Lüst and S. Stieberger, "Four-dimensional String Compactifications with D-Branes, Orientifolds and Fluxes," Phys. Rept. 445 (2007) 1 doi:10.1016/j.physrep.2007.04.003 [hep-th/0610327].

[25] D. Lüst and S. Stieberger, "Gauge threshold corrections in intersecting brane world models," Fortsch. Phys. 55 (2007) 427 [arXiv:hep-th/0302221].

[26] N. Akerblom, R. Blumenhagen, D. Lüst and M. Schmidt-Sommerfeld, "Thresholds for Intersecting D-branes Revisited,” Phys. Lett. B 652 (2007) 53 doi:10.1016/j.physletb.2007.06.060 [arXiv:0705.2150 [hep-th]].

[27] M. Berg, M. Haack and J. U. Kang, “One-Loop Kähler Metric of D-Branes at Angles,” JHEP 1211 (2012) 091 [arXiv:1112.5156 [hep-th]].

[28] I. Antoniadis, E. Gava and K. S. Narain, "Moduli corrections to gravitational couplings from string loops,” Phys. Lett. B 283 (1992) 209 doi:10.1016/0370-2693(92)90009-S [hep-th/9203071].

[29] E. Kiritsis and C. Kounnas, "Infrared regularization of superstring theory and the one loop calculation of coupling constants,” Nucl. Phys. B 442 (1995) 472 doi:10.1016/0550-3213(95)00156-M [hep-th/9501020]. 
[30] E. Kiritsis, C. Kounnas, P. M. Petropoulos and J. Rizos, "On the heterotic effective action at one loop gauge couplings and the gravitational sector," hep-th/9605011.

[31] M. B. Green and J. H. Schwarz, "Supersymmetrical Dual String Theory. 3. Loops and Renormalization,” Nucl. Phys. B 198 (1982) 441. doi:10.1016/0550-3213(82)90334-0

[32] D. J. Gross and E. Witten, “Superstring Modifications of Einstein's Equations,” Nucl. Phys. B 277 (1986) 1. doi:10.1016/0550-3213(86)90429-3

[33] M. T. Grisaru, A. E. M. van de Ven and D. Zanon, “Two-Dimensional Supersymmetric Sigma Models on Ricci Flat Kähler Manifolds Are Not Finite,” Nucl. Phys. B 277 (1986) 388. doi:10.1016/0550-3213(86)90448-7

[34] Y. Cai and C. A. Nunez, "Heterotic String Covariant Amplitudes and Low-energy Effective Action," Nucl. Phys. B 287 (1987) 279. doi:10.1016/0550-3213(87)90106-4

[35] D. J. Gross and J. H. Sloan, “The Quartic Effective Action for the Heterotic String," Nucl. Phys. B 291 (1987) 41. doi:10.1016/0550-3213(87)90465-2

[36] N. Sakai and Y. Tanii, “One Loop Amplitudes and Effective Action in Superstring Theories,” Nucl. Phys. B 287 (1987) 457. doi:10.1016/0550-3213(87)90114-3

[37] M. Abe, H. Kubota and N. Sakai, "Loop Corrections to the Heterotic String Effective Lagrangian," Phys. Lett. B 200 (1988) 461 Addendum: [Phys. Lett. B 203 (1988) 474]. doi:10.1016/0370-2693(88)90152-9

[38] M. Abe, H. Kubota and N. Sakai, "Loop Corrections to the $E(8)$ X E(8) Heterotic String Effective Lagrangian,” Nucl. Phys. B 306 (1988) 405. doi:10.1016/0550-3213(88)90699-2

[39] M. B. Green and A. Rudra, “Type I/heterotic duality and M-theory amplitudes,” JHEP 1612 (2016) 060 doi:10.1007/JHEP12(2016)060 [arXiv:1604.00324 [hep-th]].

[40] M. Berg, M. Haack and B. Körs, "Loop corrections to volume moduli and inflation in string theory," Phys. Rev. D 71 (2005) 026005 [hep-th/0404087].

[41] S. Kachru, R. Kallosh, A. D. Linde, J. M. Maldacena, L. P. McAllister and S. P. Trivedi, "Towards inflation in string theory," JCAP 0310 (2003) 013 doi:10.1088/1475-7516/2003/10/013 [hep-th/0308055].

[42] N. Akerblom, R. Blumenhagen, D. Lüst and M. Schmidt-Sommerfeld, "Instantons and Holomorphic Couplings in Intersecting D-brane Models," JHEP 0708 (2007) 044 doi:10.1088/1126-6708/2007/08/044 [arXiv:0705.2366 [hep-th]].

[43] P. G. Camara, C. Condeescu and E. Dudas, "Holomorphic variables in magnetized brane models with continuous Wilson lines,” JHEP 1004 (2010) 029 doi:10.1007/JHEP04(2010)029 [arXiv:0912.3369 [hep-th]].

[44] I. Antoniadis, R. Minasian, S. Theisen and P. Vanhove, "String loop corrections to the universal hypermultiplet," Class. Quant. Grav. 20 (2003) 5079 doi:10.1088/0264-9381/20/23/009 [hep-th/0307268].

[45] J. P. Derendinger, S. Ferrara, C. Kounnas and F. Zwirner, "On loop corrections to string effective field theories: Field dependent gauge couplings and sigma model anomalies," Nucl. Phys. B 372 (1992) 145. doi:10.1016/0550-3213(92)90315-3

[46] P. Adamietz, P. Binetruy, G. Girardi and R. Grimm, "Supergravity and matter: Linear multiplet couplings and Kähler anomaly cancellation,” Nucl. Phys. B 401 (1993) 257. doi:10.1016/0550-3213(93)90305-9 
[47] J. P. Derendinger, F. Quevedo and M. Quiros, “The Linear multiplet and quantum four-dimensional string effective actions,” Nucl. Phys. B 428 (1994) 282 doi:10.1016/0550-3213(94)90203-8 [hep-th/9402007].

[48] M. Bianchi and A. Sagnotti, "The Partition Function of the SO(8192) Bosonic String," Phys. Lett. B 211 (1988) 407. doi:10.1016/0370-2693(88)91884-9 